\title{
HECKE MODULAR FORMS \\ AND THE KAC-PETERSON IDENTITIES
}

BY

GEORGE E. ANDREWS ${ }^{1}$

\begin{abstract}
ABSTRACr. The identity of certain Hecke modular forms with well-known infinite products is derived in an elementary manner. New identities and applications are discussed.
\end{abstract}

1. Introduction. Hecke appears to have been the first to consider extensively theta series related to indefinite quadratic forms [5]. Namely, Hecke considered series of the form

$$
\sum_{(n, m) \in D}( \pm 1)^{f(n, m)} q^{Q(n, m)+L(n, m)},
$$

where $Q$ is an indefinite quadratic form, $L$ is a linear form and $D$ is a subset of the integer points in the plane for which $Q(n, m) \geqslant 0$. Hecke was primarily interested in utilizing the algebraic number theory of quadratic fields to establish that many such series included by (1.1) are in fact modular forms. For example, Hecke showed that [5, Equation (7), p. 425]

$$
\prod_{n=1}^{\infty}\left(1-q^{n}\right)^{2}=\sum_{\substack{m, n=-\infty \\ n \geqslant 2|m|}}^{\infty}(-1)^{n+m} q^{\left(n^{2}-3 m^{2}\right) / 2+(n+m) / 2} .
$$

D. Bressoud has pointed out to me that L. J. Rogers [8, p. 323] also found (1.2); however, Rogers did not make a detailed study of such results.

Subsequently, Kac and Peterson [7], motivated by the deep and extensive affine Lie algebra machinery developed by Kac [6], showed that numerous results similar to (1.2) and including (1.2) may be proved by examining certain modular functions called string functions which arise in the analysis of the character of a highest weight representation of an affine Lie algebra. For example, Kac and Peterson show that [7, final equation]

$$
\prod_{n=1}^{\infty}\left(1-q^{n}\right)\left(1-q^{2 n}\right)=\sum_{\substack{m, n=-\infty \\ n \geqslant 3|m|}}^{\infty}(-1)^{n} q^{\left(n^{2}-8 m^{2}\right) / 2+n / 2} .
$$

Received by the editors July 26, 1982 and, in revised form, December 15, 1982.

1980 Mathematics Subject Classification. Primary 10D12; Secondary 10A45.

'Partially supported by National Science Foundation Grant MCS-8201733. 
In reality, the results (1.2) and (1.3) are immensely elegant identities for certain functions that are quite familiar in the classical theory of elliptic theta functions and $q$-series [9, Chapter $3 ; \mathbf{1}]$.

The object of this paper is to show that results such as (1.2) and (1.3) may be directly deduced from the classical theory of $q$-series [9, Chapter $3 ; 1$ ] . Indeed all such results appear to be corollaries of the following two elementary lemmas concerning the classical theta functions.

LeMma 1. For $1<|z|<|q|^{-1},|q|<1$,

$$
\prod_{n=1}^{\infty} \frac{\left(1-q^{n}\right)^{2}}{\left(1-z q^{n}\right)\left(1-z^{-1} q^{n-1}\right)}=\sum_{\substack{N, r=-\infty \\ r \geqslant|N|}}^{\infty}(-1)^{r+N} z^{N} q^{\left(r^{2}-N^{2}\right) / 2+(r+N) / 2} .
$$

LEMMA 2. Viewed as a function of $z$ in the annulus $1<|z|<|q|^{-1}$ (with $|q|<1$ and $A, B, C$ positive integers),

$$
\prod_{n=1}^{\infty} \frac{\left(1-q^{n}\right)^{2}\left(1-q^{B n}\right)\left(1-z^{A} q^{B(n-1)+C}\right)\left(1-z^{-A} q^{B n-C}\right)}{\left(1-z q^{n}\right)\left(1-z^{-1} q^{n-1}\right)}
$$

has as its constant term (i.e. the coefficient of $z^{0}$ in the Laurent series expansion of (1.5) in the annulus $1<|z|<|q|^{-1}$ )

$$
\sum_{\substack{j, r=-\infty \\ r \geq A|j|}}^{\infty}(-1)^{r+(A+1) j} q^{\left(\left(B-A^{2}\right) j^{2}+r^{2}\right) / 2+((B+A-2 C) j+r) / 2} .
$$

We shall prove these lemmas in $\S 2$. In $\S 3$ we illustrate how to use Lemma 2 to derive (1.2), (1.3) and presumably all similar results.

2. The two lemmas. We begin by proving Lemma 1 . The classical partial fractions decomposition of the left side of (1.4) [10, p. 136] is

$$
\prod_{n=1}^{\infty} \frac{\left(1-q^{n}\right)^{2}}{\left(1-z q^{n}\right)\left(1-z^{-1} q^{n-1}\right)}=\sum_{r=0}^{\infty} \frac{(-1)^{r} q^{\left(r^{2}+r\right) / 2}}{1-z^{-1} q^{r}}-z \sum_{r=1}^{\infty} \frac{(-1)^{r} q^{\left(r^{2}+r\right) / 2}}{1-z q^{r}} .
$$

Hence for $1<|z|<|q|^{-1}$,

$$
\begin{aligned}
& \prod_{n=1}^{\infty} \frac{\left(1-q^{n}\right)^{2}}{\left(1-z q^{n}\right)\left(1-z^{-1} q^{n-1}\right)}=\sum_{r=0}^{\infty}(-1)^{r} q^{\left(r^{2}+r\right) / 2} \sum_{N=0}^{\infty} z^{-N} q^{r N} \\
& +\sum_{r=0}^{\infty}(-1)^{r} q^{\left(r^{2}+r\right) / 2} \sum_{N=1}^{\infty} z^{N} q^{N(r+1)} \\
& =\sum_{N=0}^{\infty} z^{-N} \sum_{r=N+1}^{\infty}(-1)^{r+N+1} q^{\left(r^{2}-N^{2}\right) / 2-(r+N) / 2} \\
& +\sum_{N=1}^{\infty} z^{N} \sum_{r=N+1}^{\infty}(-1)^{r+N+1} q^{\left(r^{2}-N^{2}\right) / 2-(r-N) / 2}
\end{aligned}
$$




$$
\begin{aligned}
& =\sum_{N=-\infty}^{0} z^{N} \sum_{r=|N|+1}^{\infty}(-1)^{r+N+1} q^{\left(r^{2}-N^{2}\right) / 2-(r-N) / 2} \\
& +\sum_{N=1}^{\infty} z^{N} \sum_{r=|N|+1}^{\infty}(-1)^{r+N+1} q^{\left(r^{2}-N^{2}\right) / 2-(r-N) / 2} \\
& =\sum_{\substack{N=-\infty \\
r>|N|}}^{\infty}(-1)^{r+N+1} z^{N} q^{\left(r^{2}-N^{2}\right) / 2-(r-N) / 2} \\
& =\sum_{\substack{N=-\infty \\
r \geqslant|N|}}^{\infty}(-1)^{r+N} z^{N} q^{\left(r^{2}-N^{2}\right) / 2+(r+N) / 2} .
\end{aligned}
$$

Thus Lemma 1 is established.

Lemma 2 is an almost immediate corollary of Lemma 1. First we note that (2.2)

$$
\sum_{j=-\infty}^{\infty}(-1)^{j} z^{A j} q^{B\left(\begin{array}{l}
1 \\
2
\end{array}\right)+C j}=\prod_{n=1}^{\infty}\left(1-q^{B n}\right)\left(1-z^{A} q^{B(n-1)+C}\right)\left(1-z^{-A} q^{B n-C}\right),
$$

by Jacobi's triple product identity [2, Theorem 2.8, p. 21] (where $\left(\begin{array}{l}j \\ 2\end{array}\right)=j(j-1) / 2$ and $z \neq 0,|q|<1)$. The quantity in (1.5) is the product of the right side of $(2.2)$ and the left side of (1.4). Hence the desired constant term is the coefficient of $z^{0}$ in

$$
\sum_{j=-\infty}^{\infty}(-1)^{j} z^{A j} q^{B\left(\frac{1}{2}\right)+C j} \sum_{\substack{N, r=-\infty \\ r \geqslant|N|}}^{\infty}(-1)^{r+N} z^{N} q^{\left(r^{2}-N^{2}\right) / 2+(r+N) / 2} .
$$

One now obtains all contributions to $z^{0}$ by deleting every term in the sum over $N$ except $N=-A j$. The resulting expression is precisely the one given in (1.6).

3. The identities. Let us begin with (1.2). From Lemma 2 (with $B=1, A=2$, $C=1$ ) we see that the right side of (1.2) is the constant term in

$$
\prod_{n=1}^{\infty} \frac{\left(1-q^{n}\right)^{3}\left(1-z^{2} q^{n}\right)\left(1-z^{-2} q^{n-1}\right)}{\left(1-z q^{n}\right)\left(1-z^{-1} q^{n-1}\right)}
$$

Now by the quintuple product identity [1, Theorem 3.9, p. 466; 9, Equation (7.4.7), p. 205], we know that the Laurent series expansion of the expression in (3.1) is

$$
\prod_{n=1}^{\infty}\left(1-q^{n}\right)^{2} \sum_{m=-\infty}^{\infty}(-1)^{m} q^{m(3 m-1) / 2} z^{-3 m}\left(1+z^{-1} q^{m}\right) .
$$

Thus the constant term in (3.1) is $\Pi_{n \geqslant 1}\left(1-q^{n}\right)^{2}$ and by Lemma 2 we have established (1.2).

The Kac-Peterson result (1.3) is somewhat more cumbersome but conceptually just as straightforward as (1.2). To make our argument simpler we prove an auxiliary result. 
LemMa 3. For $z, \alpha, \beta$ all nonzero and $|q|<1$,

$$
\begin{gathered}
\prod_{n=1}^{\infty}\left(1-z \alpha q^{n}\right)\left(1-z \beta q^{n}\right)\left(1-z^{-1} \alpha^{-1} q^{n-1}\right)\left(1-z^{-1} \beta^{-1} q^{n-1}\right) \\
=A_{0}(\alpha, \beta, q) \sum_{N=-\infty}^{\infty} q^{N^{2}+N^{N}} \alpha^{N} \beta^{2 n} \\
-A_{1}(\alpha, \beta, q) \sum_{N=-\infty}^{\infty} q^{N^{2}} \alpha^{N} \beta^{N} z^{2 N-1},
\end{gathered}
$$

where

$$
\begin{aligned}
A_{0}(\alpha, \beta, q) & =\beta A_{1}\left(\alpha q^{-1}, \beta, q\right) \\
& =\prod_{n=1}^{\infty}\left(1+q^{n}\right)\left(1-q^{n}\right)^{-1}\left(1+\alpha \beta^{-1} q^{2 n-1}\right)\left(1+\beta \alpha^{-1} q^{2 n-1}\right) .
\end{aligned}
$$

PROOF. To streamline notation we introduce the standard terminology of basic hypergeometric series [9, p. 89]:

$$
\begin{gathered}
(A ; q)_{N}=(A)_{N}=(1-A)(1-A q) \cdots\left(1-A q^{N-1}\right), \quad \text { and } \\
(A)_{\infty}=\lim _{N \rightarrow \infty}(A)_{N} .
\end{gathered}
$$

Thus the left side of (3.3) is, by Jacobi's triple product identity [2, Theorem 2.8, p. $21]$

$$
\begin{aligned}
& \frac{1}{(q)_{\infty}^{2}} \sum_{n=-\infty}^{\infty}(-1)^{n} z^{n} \alpha^{n} q^{\left(n_{2}+1\right)} \sum_{m=-\infty}^{\infty}(-1)^{m} z^{m} \beta^{m} q^{\left(m_{2}+1\right)} \\
& =\frac{1}{(q)_{\infty}^{2}} \sum_{N=-\infty}^{\infty}(-1)^{N} z^{N} q^{\left({ }^{N+1} 2^{2}\right.} \beta^{N} \sum_{n=-\infty}^{\infty} \alpha^{n} \beta^{-n} q^{n^{2}-n N} \\
& \text { (wherein } m \text { has been replaced by } N-n \text { ) } \\
& =\frac{1}{(q)_{\infty}^{2}}\left\{\sum_{N=-\infty}^{\infty} z^{2 N} q^{(2 .+2)-N^{2}} \beta^{2 N} \sum_{n=-\infty}^{\infty} \alpha^{n} \beta^{-n} q^{(n-N)^{2}}\right. \\
& \left.-\sum_{N=-\infty}^{\infty} z^{2 N-1} q^{(2 N)-N^{2}+N} \beta^{2 N-1} \sum_{n=-\infty}^{\infty} \alpha^{n} \beta^{-n} q^{(n-N)^{2}+n-N}\right\} \\
& =\frac{1}{(q)_{\infty}^{2}}\left\{\sum_{N=-\infty}^{\infty} z^{2 N} q^{N^{2}+N^{N}} \alpha^{N} \beta^{N} \sum_{n=-\infty}^{\infty} \alpha^{n} \beta^{-n} q^{n^{2}}\right. \\
& \left.-\sum_{N=-\infty}^{\infty} z^{2 N-1} q^{N^{2}} \alpha^{N} \beta^{N} \beta^{-1} \sum_{n=-\infty}^{\infty} \alpha^{n} \beta^{-n} q^{n^{2}+n}\right\} .
\end{aligned}
$$


Hence Lemma 3 is established once we show that

$$
A_{0}(\alpha, \beta, q)=\beta A_{1}\left(\alpha q^{-1}, \beta, q\right)=\frac{1}{(q)_{\infty}^{2}} \sum_{n=-\infty}^{\infty} \alpha^{n} \beta^{-n} q^{n^{2}} \text {. }
$$

The identity of the expression in (3.6) with that in (3.4) follows immediately from Jacobi's triple product identity [2, Theorem 2.8, p. 21]. Hence Lemma 3 is established.

We now turn to identity (1.3). By Lemma 2 (with $A=3, B=1, C=2$ ) we see that the right side of (1.3) is the constant term in

$$
\begin{aligned}
\prod_{n=1}^{\infty} & \frac{\left(1-q^{n}\right)^{3}\left(1-z^{3} q^{n+1}\right)\left(1-z^{-3} q^{n-2}\right)}{\left(1-z q^{n}\right)\left(1-z^{-1} q^{n-1}\right)}=\frac{(q)_{\infty}^{3}\left(z^{3} q^{2}\right)_{\infty}\left(z^{-3} q^{-1}\right)_{\infty}}{(z q)_{\infty}\left(z^{-1}\right)_{\infty}} \\
= & \frac{(q)_{\infty}^{3}\left(z^{3} q^{2} ; q^{3}\right)_{\infty}\left(z^{3} q^{3} ; q^{3}\right)_{\infty}\left(z^{3} q^{4} ; q^{3}\right)_{\infty}\left(z^{-3} q^{-1} ; q^{3}\right)_{\infty}\left(z^{-3} ; q^{3}\right)_{\infty}\left(z^{-3} q ; q^{3}\right)}{(z q)_{\infty}\left(z^{-1}\right)_{\infty}} \\
= & (q)_{\infty}^{3}\left\{\left(z^{3} q^{2} ; q^{3}\right)_{\infty}\left(z^{3} q^{4} ; q^{3}\right)_{\infty}\left(z^{-3} q ; q^{3}\right)\left(z^{-3} q^{-1} ; q^{3}\right)_{\infty}\right\}\left\{\frac{\left(z^{3} q^{3} ; q^{3}\right)_{\infty}\left(z^{-3} ; q^{3}\right)_{\infty}}{(z q)_{\infty}\left(z^{-1}\right)_{\infty}}\right\} \\
= & (q)_{\infty}^{3}\left\{\left(z^{3} q^{2} ; q^{3}\right)_{\infty}\left(z^{3} q^{4} ; q^{3}\right)_{\infty}\left(z^{-3} q ; q^{3}\right)_{\infty}\left(z^{-3} q^{-1} ; q^{3}\right)_{\infty}\right\} \\
& \times\left\{(z \omega q)_{\infty}\left(z \omega^{2} q\right)_{\infty}\left(z^{-1} \omega\right)_{\infty}\left(z^{-1} \omega^{2}\right)_{\infty}\right\} \quad\left(\text { where } \omega=e^{2 \pi i / 3}\right) \\
= & \left.(q)_{\infty}^{3}\left\{A_{0}\left(q^{-1}, q, q^{3}\right) \sum_{N=-\infty}^{\infty}(-1)^{N} q^{3 N^{2}+3 z^{6 N}}, q, q^{3}\right) \sum_{N=-\infty}^{\infty}(-1)^{N} q^{3 N^{2}} z^{6 N-3}\right\} \\
& \times\left\{A_{0}\left(\omega, \omega^{2}, q\right) \sum_{N=-\infty}^{\infty}(-1)^{N} q^{N^{2}+z_{z} 2 N}-A_{1}\left(\omega, \omega^{2}, q\right) \sum_{N=-\infty}^{\infty}(-1)^{N} q^{\left.N^{2} z^{2 N-1}\right\} .}\right.
\end{aligned}
$$

Now the constant term in this last expression is clearly

$$
\begin{aligned}
(q)_{\infty}^{3}\left\{A _ { 0 } \left(q^{-1},\right.\right. & \left.q, q^{3}\right) A_{0}\left(\omega, \omega^{2}, q\right) \sum_{N=-\infty}^{\infty} q^{12 N^{2}} \\
& \left.+A_{1}\left(q^{-1}, q, q^{3}\right) A_{1}\left(\omega, \omega^{2}, q\right) \sum_{N=-\infty}^{\infty} q^{12 N^{2}-12 N+4}\right\} .
\end{aligned}
$$

Furthermore, from (3.4), we see that

$$
\begin{gathered}
A_{0}\left(\omega, \omega^{2}, q\right)=\left(-q^{2} ; q^{2}\right)_{\infty}\left(-q^{3} ; q^{6}\right)_{\infty} /(q)_{\infty} \\
A_{0}\left(q^{-1}, q, q^{3}\right)=\left(-q^{6} ; q^{6}\right)_{\infty}\left(-q ; q^{2}\right)_{\infty} /\left(q^{3} ; q^{3}\right)_{\infty} \\
A_{1}\left(\omega, \omega^{2}, q\right)=-\left(-q ; q^{2}\right)_{\infty}\left(-q^{6} ; q^{6}\right)_{\infty} /(q)_{\infty}
\end{gathered}
$$

and

$$
A_{1}\left(q^{-1}, q, q^{3}\right)=q^{-1}\left(-q^{3} ; q^{6}\right)_{\infty}\left(-q^{2} ; q^{2}\right)_{\infty} /\left(q^{3} ; q^{3}\right)_{\infty}
$$


Consequently, the expression given by (3.7) is precisely

$$
\begin{aligned}
&(q)_{\infty}^{3}\left\{\frac{(-q)_{\infty}\left(-q^{3} ; q^{3}\right)_{\infty}}{(q)_{\infty}\left(q^{3} ; q^{3}\right)_{\infty}} \sum_{N=-\infty}^{\infty} q^{12 N^{2}}\right.\left.-q^{-1} \frac{(-q)_{\infty}\left(-q^{3} ; q^{3}\right)_{\infty}}{(q)_{\infty}\left(q^{3} ; q^{3}\right)_{\infty}} \sum_{N=-\infty}^{\infty} q^{12 N^{2}+12 N+4}\right\} \\
&= \frac{(q)_{\infty}^{2}(-q)_{\infty}\left(-q^{3} ; q^{3}\right)_{\infty}}{\left(q^{3} ; q^{3}\right)_{\infty}} \sum_{N=-\infty}^{\infty}(-1)^{N} q^{3 N^{2}} \\
&=(q)_{\infty}^{2}(-q)_{\infty}=(q)_{\infty}\left(q^{2} ; q^{2}\right)_{\infty}=\prod_{n=1}^{\infty}\left(1-q^{n}\right)\left(1-q^{2 n}\right)
\end{aligned}
$$

(by [2, Equation (2.2.12), p. 23]),

the desired left side of (1.3).

As a final example of the efficacy of our approach we establish that

$$
\begin{aligned}
& \sum_{r \geqslant 4|j|}(-1)^{r+j} q^{(r+1)-j(15 j+1) / 2} \\
& \quad=\prod_{n=1}^{\infty}\left(1-q^{n}\right)\left(1-q^{15 n}\right)\left(1-q^{15 n-6}\right)\left(1-q^{15 n-9}\right) .
\end{aligned}
$$

From Lemma 2 (with $B=1, A=4, C=3$ ) we see that the left side of (3.13) is the constant term in

$$
\begin{aligned}
\prod_{n=1}^{\infty} & \frac{\left(1-q^{n}\right)^{3}\left(1-z^{4} q^{n+3}\right)\left(1-z^{-4} q^{n-2}\right)}{\left(1-z q^{n}\right)\left(1-z^{-1} q^{n-1}\right)} \\
= & (q)_{\infty}\left\{\frac{(q)_{\infty}\left(z^{4} q^{3}\right)_{\infty}\left(z^{-4} q^{-2}\right)_{\infty}}{\left(z^{2} q^{2}\right)_{\infty}\left(z^{-2} q^{-1}\right)_{\infty}}\right\}\left\{\frac{(q)_{\infty}\left(z^{2} q\right)_{\infty}\left(z^{-2}\right)_{\infty}}{(z q)_{\infty}\left(z^{-1}\right)_{\infty}}\right\}\left(-z^{-2} q^{-1}\right) \\
= & (q)_{\infty}\left(-z^{-2} q^{-1}\right)\left\{\sum_{m=-\infty}^{\infty}(-1)^{m} q^{m(3 m-7) / 2} z^{-6 m}\left(1+z^{-2} q^{m-1}\right)\right\} \\
& \times\left\{\sum_{n=-\infty}^{\infty}(-1)^{n} q^{n(3 n-1) / 2} z^{-3 n}\left(1+z^{-1} q^{n}\right)\right\}
\end{aligned}
$$

(by the identity of (3.1) with (3.2)).

Now the constant term in this last expression arises only when $n=-2 m-1$ and then only from the second term in the first sum multiplied by the second term in the second sum. Consequently, the desired constant term is

$$
\begin{gathered}
\left(-q^{-1}\right)(q)_{\infty} \sum_{m=-\infty}^{\infty}(-1)^{m} q^{m(3 m-7) / 2}(-1)^{2 m+1} q^{\left(3(2 m+1)^{2}-(2 m+1)\right) / 2} \\
=(q)_{\infty} \sum_{m=-\infty}^{\infty}(-1)^{m} q^{m(15 m+3) / 2}
\end{gathered}
$$


and this last expression is immediately identified with the right side of (3.13) by Jacobi's triple product identity [2, Theorem 2.8 , p. 21].

Obviously, these techniques may be applied extensively. One merely chooses $A, B$ and $C$ in Lemma 2 and then appropriately factorizes the infinite product into theta series; the constant term is then read off to provide the desired identity.

Further quite straightforward examples are:

$$
\sum_{r=0}^{\infty}(-1)^{r}(2 r+1) q^{r(r+1) / 2}=\prod_{n=1}^{\infty}\left(1-q^{n}\right)^{3} \quad(A=B=C=1) ;
$$

$$
\sum_{\substack{j, r=-\infty \\ r \geqslant 2|j|}}^{\infty}(-1)^{r+j} q^{\left(r_{2}^{+1}\right)-j^{2}}=\prod_{n=1}^{\infty}\left(1-q^{n}\right)\left(1-q^{2 n}\right) \quad(A=B=C=2) ;
$$

$$
\begin{aligned}
& \sum_{\substack{j, r=-\infty \\
r \geq 3|j|}}^{\infty}(-1)^{r} q^{(r+1)-3 j^{2}} \\
& =\prod_{n=1}^{\infty} \frac{\left(1-q^{n}\right)\left(1-q^{3 n}\right)}{\left(1-q^{12 n-2}\right)\left(1-q^{12 n-3}\right)\left(1-q^{12 n-9}\right)\left(1-q^{12 n-10}\right)} \\
& =\prod_{n=1}^{\infty} \frac{\left(1-q^{n}\right)\left(1-q^{6 n}\right)}{\left(1+q^{6 n}\right)\left(1-q^{4 n-2}\right)} \quad(A=B=C=3) \text {; }
\end{aligned}
$$

$$
\begin{aligned}
& \sum_{\substack{j, r=-\infty \\
r \geq 4|j|}}^{\infty}(-1)^{r+j} q^{(r+1)-6 j^{2}} \\
& \quad=\prod_{n=1}^{\infty} \frac{\left(1-q^{4 n}\right)\left(1-q^{6 n}\right)\left(1+q^{6 n-3}\right)^{2}}{\left(1+q^{n}\right)} \quad(A=B=C=4) .
\end{aligned}
$$

Of these last four results we note that (3.16) is a famous formula of Jacobi [4, Theorem 357, p. 285]. The identities in (3.17)-(3.19) arise naturally in the theory of generalized Frobenius partitions [3]. Indeed identities (3.17)-(3.19) provide very efficient computational algorithms for generalized Frobenius partition functions.

4. Conclusion. The referee pointed out a number of important facts about the results in \$3. In particular, he asked whether (3.13) could be established by the methods of this paper. He also provided a beautiful proof of the equivalence of (1.3) and (3.16), namely:

Put $D_{x}=\frac{1}{2}(1, x)+\mathbf{Z}^{2}$, and for $(u, v) \in D_{0} \cup D_{1}$, put $\varepsilon(u, v)=(-1)^{u-2 v-1 / 2}$, $Q(u, v)=\frac{1}{2}\left(u^{2}-8 v^{2}-\frac{1}{4}\right)$. Put $F=\left\{(u, v) \in \mathbf{R}^{2} \mid u \geqslant 4 v>-u\right\}, F^{\prime}=\{(u, v) \in$ $\left.\mathbf{R}^{2} \mid u \geqslant 3 v>-u\right\}, R=\left\{(u, v) \in \mathbf{R}^{2}\left|u>8^{1 / 2}\right| v \mid\right\}$. Put $T(u, v)=(3 u+8 v, u+$ $3 v)$. The following easily-checked assertions imply the equivalence of (1.3) and (3.16):

(1) $T$ interchanges the disjoint sets $D_{0}$ and $D_{1}$ and leaves $\varepsilon$ and $Q$ invariant.

(2) $F\left(\right.$ resp. $F^{\prime}$ ) is a fundamental domain for $T$ (resp. $T^{2}$ ) on $R$. 
(3) The right-hand side of (1.3) can be written as $\sum_{\gamma \in D_{0} \cap F^{\prime}} \varepsilon(\gamma) q^{Q(\gamma)}$.

(4) The left-hand side of (3.16) can be written as $\sum_{\gamma \in\left(D_{0} \cup D_{1}\right) \cap F} \varepsilon(\gamma) q^{Q(\gamma)}$.

Since (3.16) follows trivially from Lemma 2 , the above yields a much more elegant proof of (1.3) than that given in $\S 3$. However, the method that we used to prove (1.3) is a perfect prototype of how our techniques work. Indeed everything leading up to (3.12) is quite routine in any example. In this instance great simplification occurs thereafter. If it had not (as in the cases $A=B=C>4$ ), then we would have to be content with the representation of our series as a sum of several modular forms.

\section{REFERENCES}

I. G. E. Andrews, Applications of basic hypergeometric functions, SIAM Rev. 16 (1974), 441-484.

2. Mass., 1976. , The theory of partitions, Encyclopedia of Math. Appl., vol. 2, Addison-Wesley, Reading,

3. Generalized Frobenius partitions, Mem. Amer. Math. Soc. (to appear).

4. G. H. Hardy and E. M. Wright, An introduction to the theory of numbers (4th ed.), Oxford Univ. Press, London and New York, 1960.

5. E. Hecke, Über einen Zusammenhang zwischen elliptischen Modulfunktionen und indefiniten quadratischen Formen, Mathematische Werke, Vandenhoeck and Ruprecht, Göttingen, 1959, pp. 418-427.

6. V. G. Kac, Infinite-dimensional algebras, Dedekind's $\eta$-function, classical Möbius function and the very strange formula, Adv. in Math. 30 (1978), 85-136.

7. V. G. Kac and D. H. Peterson, Affine Lie algebras and Hecke modular forms, Bull. Amer. Math. Soc. (N.S.) 3 (1980), 1057-1061.

8. L. J. Rogers, Second memoir on the expansion of certain infinite products, Proc. London Math. Soc. 25 (1894), 318-343.

9. L. J. Slater, Generalized hypergeometric functions, Cambridge Univ. Press, London and New York, 1966.

10. J. Tannery and J. Molk, Eléments de la théorie des fonctions elliptiques, Tome III, Gauthier-Villars, Paris, 1893-1902; reprinted, Chelsea, New York, 1972.

Department of Mathematics, Pennsylvania State University, University Park, Pennsylvania 16802 